\title{
PIN1 in hepatocellular carcinoma is associated with TP53 gene status
}

\author{
JUN SANG BAE ${ }^{1}$, SANG JAE NOH ${ }^{2}$, KYOUNG MIN KIM $^{1}$, KYU YUN JANG ${ }^{1}$, HO SUNG PARK ${ }^{1}$, \\ MYOUNG JA CHUNG ${ }^{1}$, BYUNG-HYUN PARK ${ }^{3}$ and WOO SUNG MOON ${ }^{1}$ \\ ${ }^{1}$ Department of Pathology, Chonbuk National University Medical School, Research Institute of Clinical Medicine \\ of Chonbuk National University-Biomedical Research Institute of Chonbuk National University Hospital \\ and Research Institute for Endocrine Sciences, Jeonju 561-756; \\ ${ }^{2}$ Department of Forensic Medicine, Chonbuk National University Medical School, Jeonju 561-756; \\ ${ }^{3}$ Department of Biochemistry, Chonbuk National University Medical School, and \\ Research Institute for Endocrine Sciences, Jeonju 561-756, Republic of Korea
}

Received March 24, 2016; Accepted July 22, 2016

DOI: 10.3892/or.2016.5001

\begin{abstract}
Phosphorylation of proteins on serine/threonine residues that precede proline (pSer/Thr-Pro) is specifically catalyzed by the peptidyl-prolyl cis-trans isomerase PIN1. PIN1-mediated prolyl-isomerization induces cell cycle arrest and growth inhibition through the regulation of target proteins, including TP53. We examined whether PIN1 acts in a different manner according to TP53 gene status in hepatocellular carcinoma (HCC). We investigated the expression of PIN1 and TP53 proteins in 119 HCC tissue samples. We also analyzed PIN1 expression in combination with TP53 gene mutation and its correlation with the clinical outcome. In addition, we used synthetic small interfering RNA to silence PINI gene expression in TP53 wild-type and TP53 mutant HCC cell lines, and then evaluated cell proliferation, migration and invasion. Expression of PIN1 was strongly associated with expression of TP53 protein or TP53 mutation of HCC samples. PIN1 and TP53 expression in TP53 mutant HCC cell lines was higher than that in TP53 wild-type HCC cell lines. Silencing of PIN1 in HLE cells containing mutant TP53 significantly decreased cell proliferation, migration and invasion. In contrast to PIN1 silencing in HLE cells, PIN1 silencing in HepG2 cells containing functional wild-type TP53 resulted in enhanced tumor cell proliferation. HCC patients bearing PIN1
\end{abstract}

Correspondence to: Professor Woo Sung Moon, Department of Pathology, Chonbuk National University Medical School, Research Institute of Clinical Medicine of Chonbuk National UniversityBiomedical Research Institute of Chonbuk National University Hospital and Research Institute for Endocrine Sciences, 567 Baekjedaero, Deokjin-gu, Jeonju-si, Jeollabuk-do, Jeonju 561-756, Republic of Korea

E-mail: mws@chonbuk.ac.kr

Key words: hepatocellular carcinoma, TP53, PIN1 expression with wild-type TP53 were predicted to demonstrate favorable relapse-free survival. Our results suggest that PIN1 plays a role in cancer cell proliferation, migration and invasion in a different manner according to the TP53 gene mutation status in HCC. In particular, interaction of PIN1 with mutant TP53 can act as a tumor promoter and increase its oncogenic activities in HCC.

\section{Introduction}

Hepatocellular carcinoma (HCC) is the sixth most prevalent human cancer and the second common cause of cancer-related death worldwide (1). Although considerable advances have been made in clinical diagnosis and management of HCC, it is still associated with a high rate of mortality and poor prognosis (2). TP53 is a tumor suppressor gene that plays important roles in cellular stress response and restrains cancer initiation and progression. TP53 mutations change TP53 protein from a tumor suppressor into an oncogene (3). TP53 mutations are among the most frequent genetic alterations in human cancer, including HCC $(4,5)$. TP53 mutations primarily occur in the DNA binding domain, resulting in disruption of tumor suppressor activity due to the inability to recognize wild-type TP53 consensus sequences. TP53 mutations also acquire new oncogenic functions through a gain of function $(3,4,6)$. TP53 protein has been detected immunohistochemically in cancer cells by virtue of its high accumulation in cell nuclei and is regarded a highly specific indicator of TP53 gene mutation (7).

Phosphorylation of proteins on serine/threonine residues that precede proline (pSer/Thr-Pro) is a main signaling mechanism controlling cell cycle regulation, differentiation and proliferation. Peptidyl-prolyl isomerase PIN1 can change the conformation of phosphoproteins and modulate the function and stability of proteins (8). Accumulating evidence has demonstrated that PIN1 is overexpressed in various human cancers and plays a critical role in the transformation of epithelial cells by activating multiple oncogenic pathways $(9,10)$. In contrast, several studies have shown that decreased levels of 
PIN1 result in cellular transformation, and restoration of PIN1 can attenuate the growth of tumor cells $(8,11,12)$. These contradictory reports regarding the function of PIN1 in oncogenesis suggest that PIN1 can either function as a conditional tumor promoter or suppressor. Among the many documented targets of PIN1-mediated prolyl-isomerization that regulate cell fate, TP53 represents the most relevant one and is frequently deregulated in cancer $(8,10,13)$. A recent study has shown that PIN1 conveys oncogenic signals in concert with mutant TP53 protein to promote aggressiveness in breast cancer cells, and concomitant high PIN1 expression and TP53 mutation have been proposed as an independent prognostic factor of poor clinical outcome in breast cancer patients (14). Based on these observations, we hypothesized that PIN1 acts in a different manner according to the TP53 gene mutation status in HCC.

In the present study, we examined: i) the relationship between PIN1 and TP53 protein expression in surgical specimens of human HCC; ii) the relationship between PIN1 expression and TP53 mutation; iii) whether PIN1 silencing by small interfering RNA (siRNA) differently affects cell growth, migration, and invasion in HCC cells according to TP53 mutation status and; iv) the association of PIN1 expression and TP53 gene mutation status with clinical outcome.

\section{Materials and methods}

Materials. The present study protocol was approved by the Institutional Review Board of Chonbuk National University Hospital. Surgical specimens of 119 formalin-fixed, paraffinembedded HCC obtained from the Surgical Pathology Archives of Chonbuk National University Hospital between 1998 and 2009 were analyzed in the present study. Patients were 25-74 years in age (mean age, 55.9) and consisted of 103 males and 16 females. A total of 91 cases were positive for hepatitis B virus surface antigen, 6 were positive for anti-hepatitis $C$ virus antibody, 10 were alcohol-related and 12 had an unknown etiology. Overall survival was calculated from the date of surgery to the date of death or the final follow-up visit. Follow-up intervals ranged from 1-194 months. To determine whether PIN1 expression is associated with TP53 mutation, we examined PIN1 protein levels in 5 HCC cell lines by western blotting. The human HCC cell lines HLE, HLF and Huh-7 containing mutant TP53 (15) were purchased from the Health Science Research Resources Bank (Osaka, Japan). The HepG2 cell line (wild-type TP53) was obtained from the American Type Culture Collection (ATCC; Manassas, VA, USA). We also used a sarcomatoid HCC cell line, designated as SH-J1 (wild-type TP53) (15). HCC cell lines were cultured according to the recommendations of the cell banks.

Immunohistochemical staining and scoring. Immunohistochemical staining for PIN1 (Santa Cruz Biotechnology, Santa Cruz, CA, USA) and TP53 (Novocastra, Newcastle, UK) was performed by a polymer intense detection system using the Bond-Max Automatic stainer (Leica Bond, Newcastle upon Tyne, UK) as previously described (16). After deparaffinization, the tissue sections were heated in a microwave oven in Target Retrieval Solution (Dako, Glostrup, Denmark) for $12 \mathrm{~min}$. The samples subjected to immunostaining were rated according to a score calculated by multiplying the area score by the intensity score of the staining. The area of staining was scored as follows: 0 ( $<10 \%$ of the cancer cells), 1 (10-29\%), $2(30-59 \%)$, or $3(\geq 60 \%)$. The intensity of the cell nuclear staining was scored as 0 (none), 1 (weak), 2 (moderate), and 3 (strong). The combined score obtained by summing the scores was used for further analysis. If the score was $\geq 3$, the tumor was considered positive; otherwise, the tumor was considered negative.

TA-cloning and DNA sequencing for TP53 gene. TA-cloning and DNA sequencing of the TP53 gene were performed as previously described (15). The RNeasy Plus Micro kit (Qiagen, Hilden, Germany) was used according to the manufacturer's protocol for extraction of total RNA from $10 \mathrm{mg}$ of frozen HCC tissue. Reverse transcription was performed using avian myeloblastosis virus reverse transcriptase (CosmoGenetech, Seoul, Korea) with an oligo(dT) primer supplied by the RT PreMix kit. The primer set for amplification of a human TP53 cds was designed according to GenBank NM_000546, using forward primer, 5'-ATGGAGGAGCCGCAGTCAGATC CTAGCGTCGAG-3' and reverse primer, 5'-TCAGTCTGAGT CAGGCCCTTTTCTGTCTTGAA-3'. PCR conditions were $95^{\circ} \mathrm{C}$ for $45 \mathrm{sec}, 60^{\circ} \mathrm{C}$ for $45 \mathrm{sec}$, and $72^{\circ} \mathrm{C}$ for $90 \mathrm{sec}$ for 35 cycles using LaboPass Pfu polymerase (CosmoGenetech). PCR products of human TP53 were purified using a LaboPass PCR purification kit (CosmoGenetech) and cloned into a pCR2.1 vector (Invitrogen, Carlsbad, CA, USA). We obtained 5-18 clones for each individual sample and attempted to sequence as many clones as possible using a BigDye Terminator Cycle Sequencing Ready Reaction kit with an ABI PRISM 3730xl Genetic Analyzer (both from Applied Biosystems, Foster City, CA, USA).

Small interfering RNA (siRNA) transfection. For siRNA transfection, PIN1 siRNA and negative control siRNA duplexes were synthesized by Bioneer Corporation (Daejeon, Korea). The PIN1 duplex had the forward and reverse sequences: 5'-CCAUUUGAAGACGCCUCGU-3' and 5'-ACGAGGCGU CUUCAAAUGG-3', respectively; and the negative control duplex specific had the forward and reverse sequences: 5 '-CCU ACGCCACCAAUUUCGU-3' and 5'-ACGAAAUUGGUGGC GUAGG-3'. The specific PIN1 siRNA or negative control siRNA was transfected using Lipofectamine RNAiMAX (Invitrogen) according to the manufacturer's instructions. All experiments were performed at $48 \mathrm{~h}$ after transfection.

Enzymatic assay for PIN1. The level of PIN1 activity was determined using the SensoLyte ${ }^{\circledR}$ Green PIN1 Activity Assay kit Fluorimetric (AnaSpec, Inc., Fremont, CA, USA), according to the manufacturer's protocols. Briefly, PIN1 substrate solution was added to HCC cell extracts and incubated. Then, the fluorescein signal was read using a Multi-Mode Microplate Reader System (Perkin-Elmer, Waltham, MA, USA) at excitation and emission wavelengths of 490 and $520 \mathrm{~nm}$, respectively.

Western blotting. Western blotting was performed as previously described (17). Briefly, the cells were collected, and pellets were lysed with PRO-PREP protein extraction solution (iNtRON Biotechnology, Inc., Seoul, Korea) containing $1 \mathrm{X}$ phosphatase inhibitor cocktails 2 and 3 (Sigma, St. Louis, MO, USA). The 

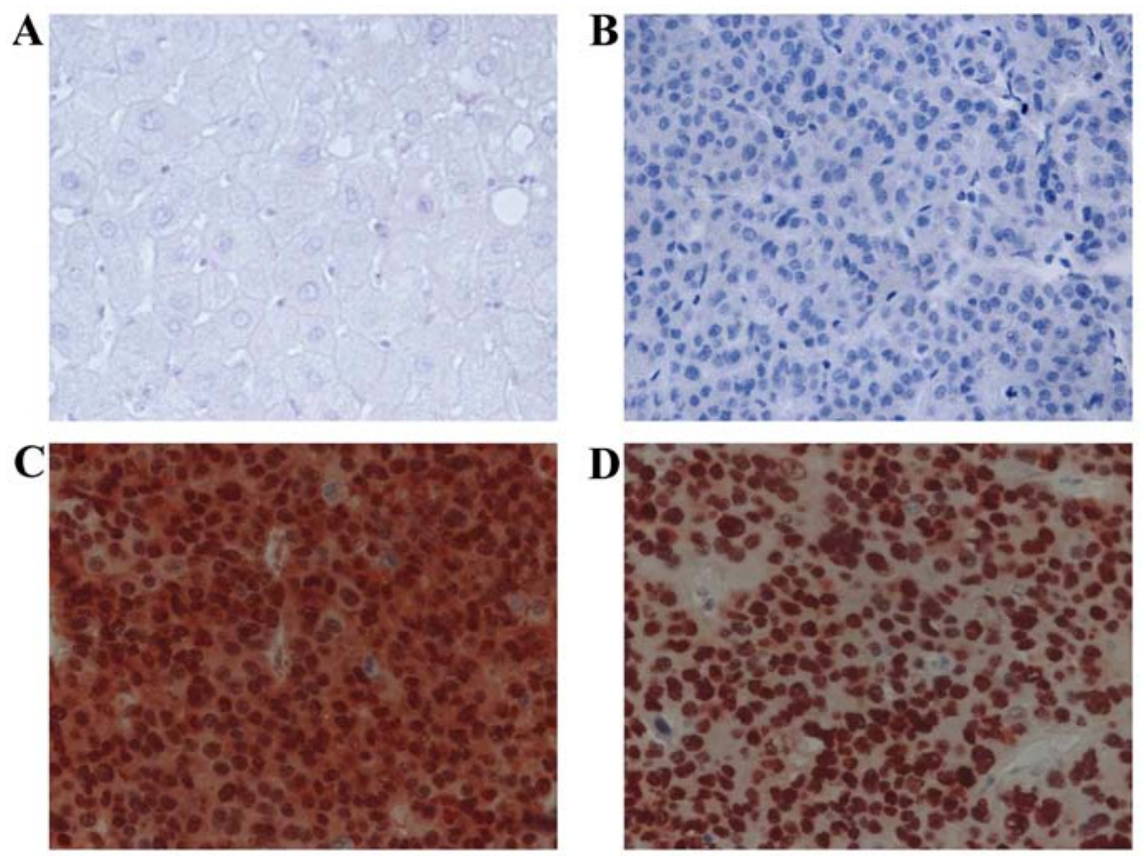

Figure 1. Immunohistochemical staining for PIN1 and TP53 protein. (A) Non-malignant hepatocytes showed no immunoreactivity for PIN1. (B) No immunoreactivity for PIN1 in HCC cells. (C) Both nuclear and cytoplasmic immunoreactivity for PIN1 in HCC cells. (D) The expression of TP53 was mainly localized in the nuclei of HCC cells.

proteins were separated by $10 \%$ SDS-polyacrylamide gel, transferred to a polyvinylidene difluoride (PVDF) membrane (Thermo Fisher Scientific, Scotts Valley, CA, USA), and probed with primary antibodies for PIN1 (Santa Cruz Biotechnology), TP53 (Novocastra) and $\beta$-actin (Sigma).

Cell proliferation assay. The cell proliferation ability of PIN1 was determined by 3-(4,5-dimethylthiazol-2-yl)-2,5-diphenyltetrazolium bromide (MTT) assay (Sigma). Post-transfection, the HepG2 or HLE cells were re-seeded in 96-well plates at 5 or $3 \times 10^{3}$ cells/well and incubated for different times (24, 48 and $72 \mathrm{~h}$ ). The absorbance of all samples was measured at $560 \mathrm{~nm}$.

In vitro migration and invasion assays. A 24-Transwell migration assay (Corning Life Sciences, Acton, MA, USA) was performed to evaluate the migration ability of the cells. The invasion assay was performed using the Transwell bioCoat Matrigel Invasion chamber (BD Biosciences). The cells that migrated to or invaded the lower surface of the filter were counted in 5 microscopic fields (magnification, x100)/well.

Statistical analysis. To evaluate the values between the groups, Pearson's Chi-square test and Student's t-test were used. P-values $<0.05$ were considered to be statistically significant. All experiments were repeated a minimum of 3 times, and representative data are presented. Survival analyses were performed using the Kaplan-Meier method, and differences in survival between different groups were determined by the log-rank test.

\section{Results}

Relationship between PIN1 and TP53 protein expression. In HCC cells, PIN1 expression was predominantly localized
Table I. Correlation between PIN1 and TP53.

A, Correlation between PIN1 and TP53 protein expression

\begin{tabular}{llccc}
\hline & & \multicolumn{2}{c}{ TP53 expression } & \\
\cline { 3 - 4 } & & Positive & Negative & P-value \\
\hline \multirow{2}{*}{ PIN1 expression } & Positive & 45 & 32 & 0.002 \\
& Negative & 12 & 30 & \\
\hline
\end{tabular}

B, Correlation between PIN1 expression and TP53 mutation

\begin{tabular}{llrcc}
\hline & & \multicolumn{2}{c}{ TP53 mutation } & \\
\cline { 3 - 4 } & & Mutation & Wild-type & P-value \\
\hline \multirow{2}{*}{ PIN1 expression } & Positive & 19 & 8 & 0.03 \\
& Negative & 5 & 9 & \\
\hline
\end{tabular}

in the nucleus. Various tumor cells showed both nuclear and cytoplasmic expression. The expression of TP53 was also mainly localized in the nuclei of tumor cells. Adjacent benign hepatocytes showed minimal or no immunoreactivity for PIN1 and TP53 (Fig. 1). Expression of PIN1 and TP53 was observed in 77 of $119(64.7 \%)$ and 57 of $119(47.9 \%)$ HCC tissues, respectively. There was a significant correlation between expression of PIN1 and TP53 in HCC tissues ( $\mathrm{P}=0.002)$ (Table IA). The expression levels of PIN1 and TP53 proteins were higher in the Huh-7, HLE and HLF cell lines containing mutant TP53 than in the HepG2 and SH-J1 cells containing wild-type TP53 (Fig. 2A). In contrast, the enzymatic activity of PIN1 
A

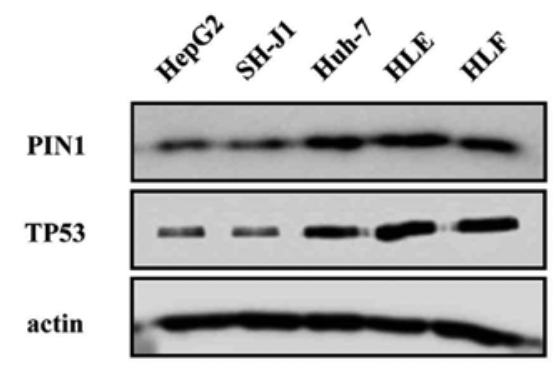

B

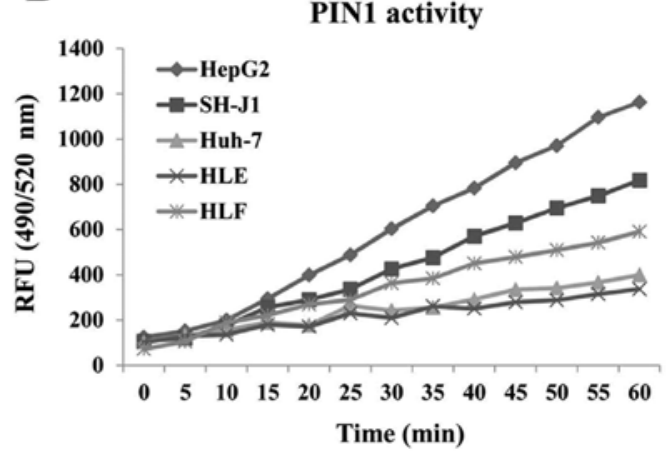

Figure 2. Western blot analysis and enzymatic activity of PIN1 in 5 different hepatocellular carcinoma cell lines. (A) The protein expression levels of PIN1 and TP53 protein were higher in Huh-7, HLE and HLF cell lines than in HepG2 and SH-J1 cells. (B) In contrast, the enzymatic activity of PIN1 was higher in the HepG2 and SH-J1 cells compared to that of HCC cell lines containing mutant TP53.
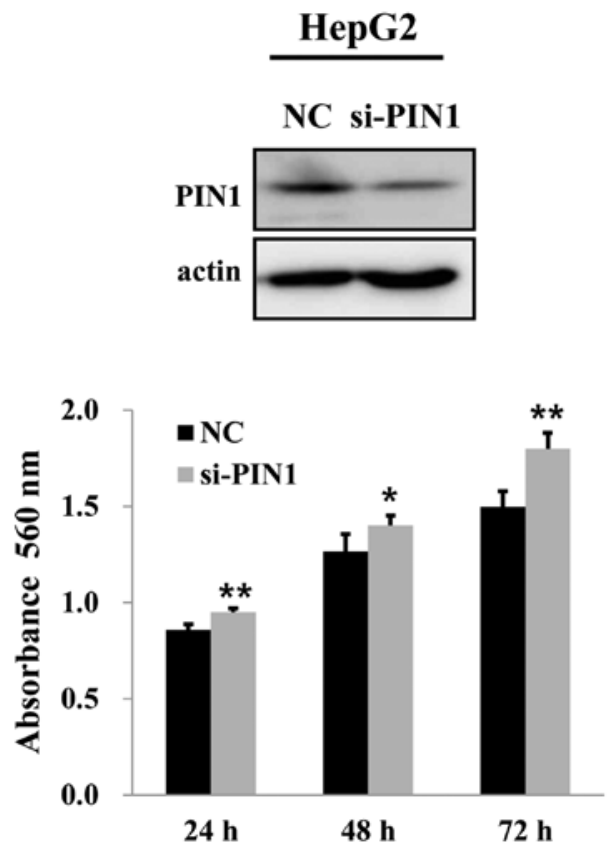

HLE

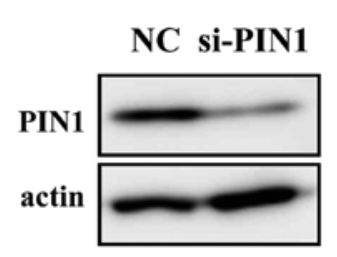

Figure 3. The effects of PIN1 silencing on HCC cell growth. PIN1 silencing in HepG2 cells increased the cell growth compared to that of the control. On the contrary, silencing of PIN1 resulted in significant decrease of cell growth in HLE cells $\left(\right.$ " $\left.\mathrm{P}<0.05,{ }^{* * *} \mathrm{P}<0.005,{ }^{*} \mathrm{P}<0.001\right)$.

was higher in the HepG2 and SH-J1 cells compared to that of HCC cell lines containing mutant TP53 (Fig. 2B).

Relationship between PIN1 expression and TP53 mutation. Positive immunostaining of TP53 is considered to be indicative of mutation or overexpression in response to cellular stress. PIN1 expression correlated with TP53 expression in our immunohistochemical study. To verify and confirm these immunohistochemical observations, we examined the relationship between PIN1 expression and TP53 mutation in HCC tissues using TP53 DNA sequencing. Forty-three frozen tissues were selected based on matching with the paraffin-embedded specimens using the immunohistochemical study. Of the 43 HCC frozen tissues, 26 had a mutation in the TP53 gene. The majority of mutations were single-nucleotide substitutions (point mutations). Only 2 HCC tissues showed an insertion mutation. The mutations in TP53 occurred predominantly in the hot-spot region (exon 5-8) [29 of 64) (45.3\%) of TP53 mutations]. Twenty-four HCCs bearing a TP53 point mutation (missense mutation) were included in the analysis of the relationship between PIN1 expression and TP53 mutation, since strong diffuse nuclear staining for TP53 antibody correlates with a missense mutation $(4,6)$. In agreement with results of immunohistochemistry, the expression of PIN1 was significantly correlated with TP53 mutation in HCC tissues $(\mathrm{P}=0.03)$ (Table IB). These results indicate that there is a good correlation between immunohistochemical expression and DNA sequencing of TP53 in determining the relationship between PIN1 expression and TP53 mutation in HCC tissues.

Effects of PIN1 silencing on cell proliferation, migration and invasion. PIN1 silencing by PIN1 siRNA in HepG2 cells containing wild-type TP53 increased the cell growth compared to that of the control. On the contrary, silencing of PIN1 resulted in a significant decrease in cell growth in HLE cells with mutant TP53 (Fig. 3). Silencing of PIN1 in HepG2 

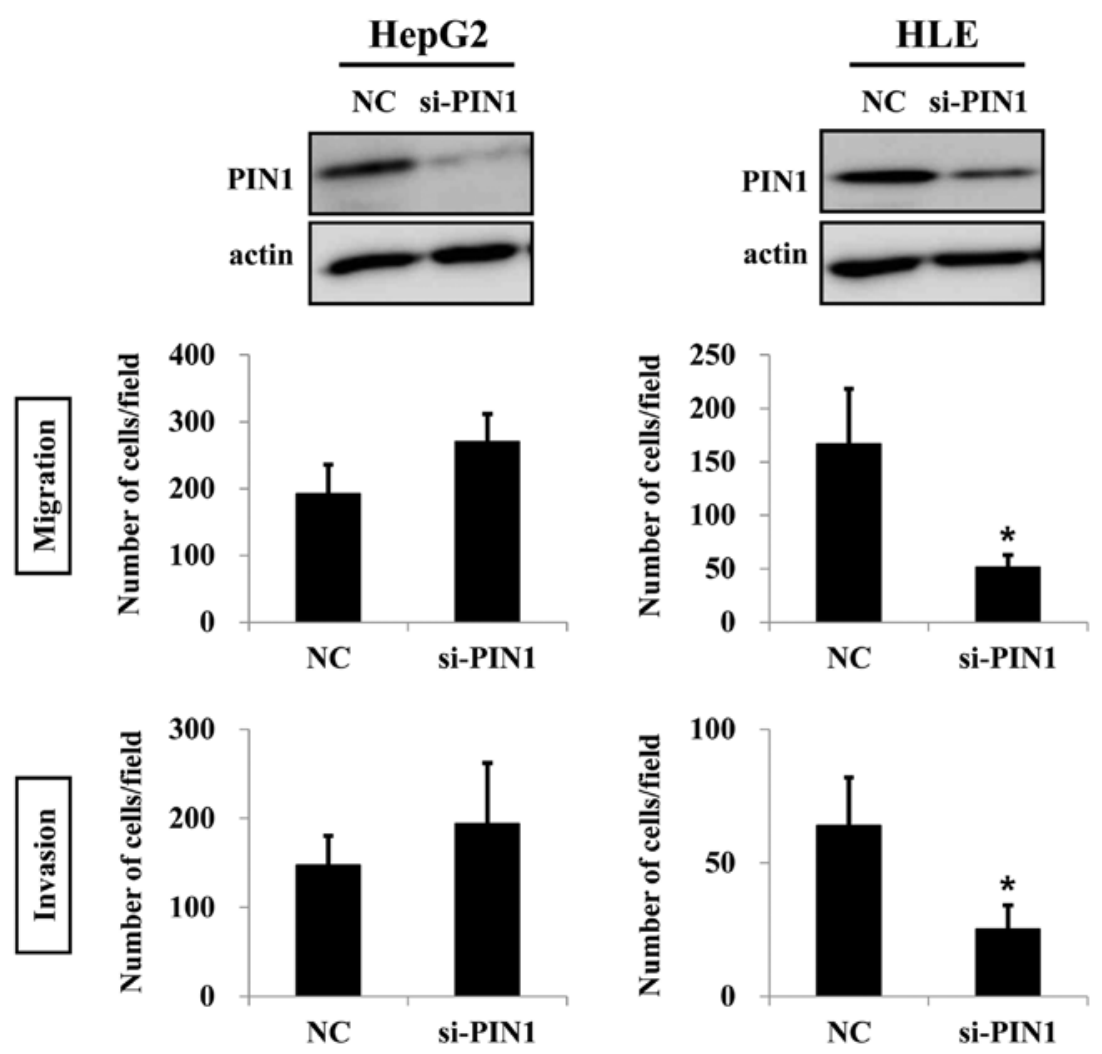

Figure 4. In vitro cell migration and invasion assay. PIN1 silencing effectively suppressed the migration and invasion of HLE cells. On the contrary, PIN1 silencing in HepG2 cells slightly increased cell migration and invasion $(" \mathrm{P}<0.05)$.

cells caused increases in migration and invasion. However, these results were not statistically significant. On the contrary, silencing of PIN1 significantly suppressed migration and invasion of HLE cells (Fig. 4).

Clinical relevance of PIN1 expression and TP53 mutation. We next examined the association of PIN1 expression and TP53 gene mutation status with clinical outcome in 43 TP53 DNA-sequenced HCC patients. The mean overall and relapse-free survival durations of patients were 71.2 and 65.6 months, respectively, for PIN1-positive HCC and 73.5 and 48.4 months for PIN1-negative HCC. PIN1 expression was associated with favorable relapse-free survival (RFS) independent of TP53 mutation status $(\mathrm{P}=0.03)$. When we determined the RFS using a putative combination of PIN1 expression with TP53 mutation status, we found that RFS was significantly longer in patients with HCCs bearing wild-type TP53 and PIN1 expression compared to that in PIN1-negative HCC patients, according to Kaplan-Meier survival analysis ( $\mathrm{P}=0.018)$ (Fig. 5). However, we did not find a significant effect on overall survival (OS) or RFS in patients with HCCs bearing mutant TP53 according to PIN1 expression status.

\section{Discussion}

Frequent high expression of PIN1 in various human cancers strongly implicates PIN1 in carcinogenesis of many different cancer types, including HCC $(8-10,18-21)$. PIN1 is critically involved in hepatocarcinogenesis via accumulation of $\beta$-catenin and cyclin D (19), or interaction with HBx in hepa- titis B virus-related HCC (20). PIN1 also facilitates NF- $\mathrm{B}$ activation and promotes tumor progression in HCC (21). However, it remains controversial whether PIN1 acts as a tumor promoter $(10,14,18-20)$ or tumor suppressor $(11,12,22)$. TP53 is one of the more than 50 critical regulatory proteins catalyzed by PIN1 $(8,13)$. TP53 mutation is also one of the most common genetic alterations in HCC and leads to the accumulation of mutant TP53 protein that endows oncogenic activities $(5,7,23)$. It has been proposed that the PIN1/mutant TP53 axis promotes aggressiveness in breast cancer (14). Accordingly, the role of PIN1 expression in conjugation with TP53 mutation status in human malignancy needs to be investigated. However, there have been no studies on the relationship between PIN1 expression with respect to TP53 gene status and its roles in HCC.

The present study is the first to demonstrate the following: i) there is a significant correlation between immunohistochemical expression of PIN1 and TP53 protein in HCC; ii) expression of PIN1 is strongly associated with TP53 mutation in HCC; iii) PIN1 and TP53 expression in TP53 mutant HCC cell lines is higher compared to that in TP53 wild-type HCC cell lines; in contrast, the enzymatic activity of PIN1 is higher in HCC cells with wild-type TP53; iv) PIN1 silencing effectively reduces tumor cell proliferation, but also cell migration and invasion capacity in HLE cells containing mutant TP53 gene. In contrast to PIN1 silencing in HLE cells, PIN1 silencing in HepG2 cells containing functional wild-type TP53 yields different results: PIN1 silencing enhances tumor cell proliferation, migration and invasion; and v) patients bearing wild-type TP53 with PIN1 expression show favorable relapse-free survival. These 

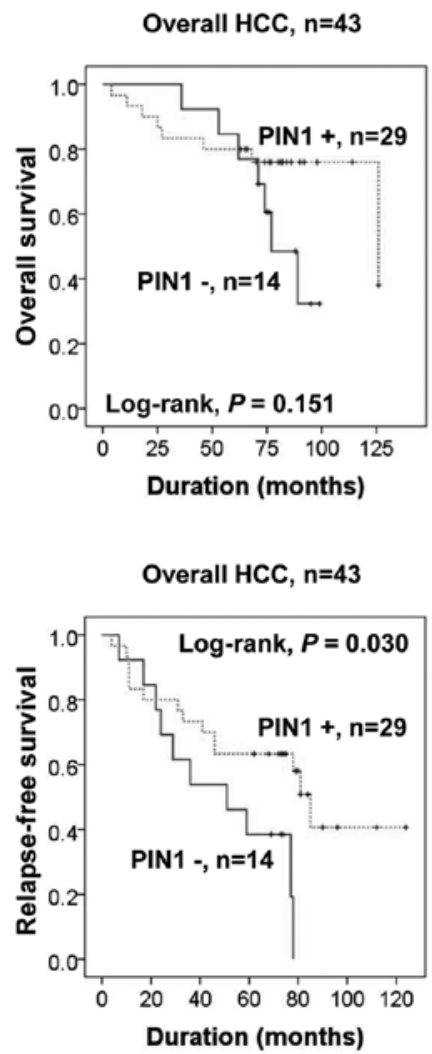

HCC, Wild-type TP53, $n=17$

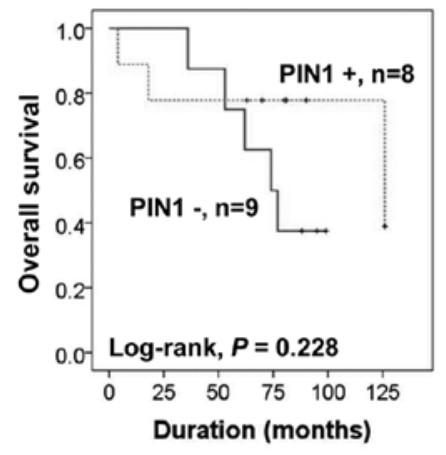

HCC, Wild-type TP53, n=17

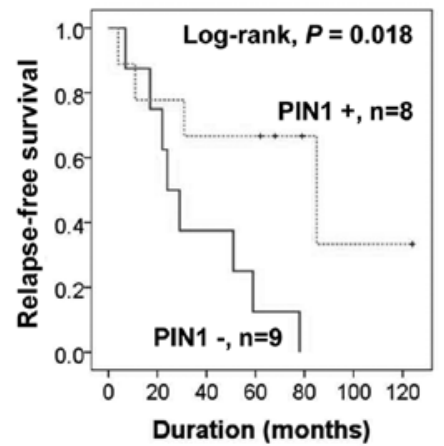

HCC, Mutant TP53, $n=26$

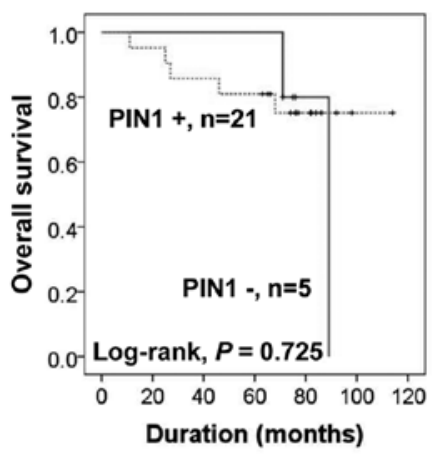

HCC, Mutant TP53, n=26

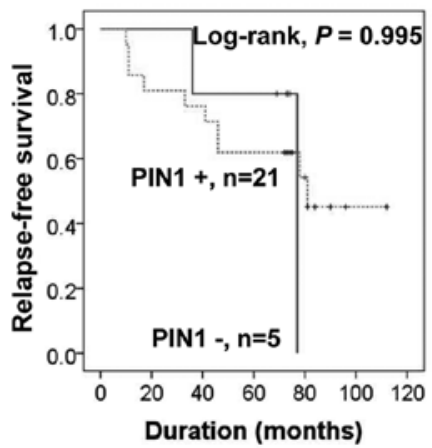

Figure 5. PIN1 expression was associated with favorable relapse-free survival independent of TP53 mutation status. RFS was significantly higher in patients with HCCs bearing wild-type TP53 with PIN1 expression than in PIN1-negative HCC patients.

findings strongly suggest that a functional interaction between PIN1 and TP53 produces different biologic outputs in tumor cell growth, migration and invasion depending on TP53 gene mutation status.

TP53 is a tumor suppressor that initiates cell cycle arrest, apoptosis, and senescence in response to cellular stress $(3,4,6)$. The isomerization of TP53 protein by PIN1 results in an alteration of protein structure/or function, which is often coupled to the stabilization of the TP53 protein $(8-10)$. We found a correlation between expression of PIN1 and TP53 protein or TP53 mutation in HCC. These observations are consistent with those of previous studies, which have reported a correlation between high expression level of PIN1 and high levels of TP53 protein in non-small cell lung and esophageal cancer $(24,25)$. Since PIN1 has been shown to stabilize wild-type TP53 (8-10), these observations suggest a possible role of PIN1 in stabilization of mutant TP53. In the present study, transfection with PIN1 siRNA in HepG2 cells containing wild-type TP53 caused an increase in cell growth; these findings are consistent with the physiologic role of PIN1, which induces cell cycle arrest and growth inhibition through interaction with wild-type TP53. This observation suggests that PIN1 functions as a tumor suppressor in conditions of intact TP53 signaling. In contrast, silencing of PIN1 resulted in a significant decrease in cell growth in HLE cells. This observation is in agreement with results from previous studies showing that RNAi-mediated PIN1 suppression inhibits HCC cell proliferation using PLC/PRF/5 and Huh-7 cell lines, which have a mutant TP53 gene $(18,21)$. It is noteworthy, that the underexpression of PIN1 is frequently seen in human cancers where the TP53 mutation is rare, including kidney and skin cancer $(9,12,22)$. PIN1 negatively influences the growth of human clear cell renal cell carcinoma (ccRCC), and this suppressive ability may be dependent on the presence of functional TP53 (12). Collectively, these observations support the notion that PIN1 can either function as a tumor promoter or suppressor depending on the genetic context. In particular, interaction of PIN1 with mutant TP53 promotes its oncogenic activities in TP53 mutant HCC. However, PIN1 may have a tumor inhibitory role in the presence of functional wild-type TP53.

Several studies have indicated that, in addition to the role of PIN1 in oncogenesis, its expression is also associated with cancer cell migration and invasion. Downregulation of PIN1 expression significantly reduces tumor cell migration and invasion in various types of cancers occurring in the breast (14), lung (26) and prostate (27), where the TP53 mutation is frequent. Consistent with these observations, we found that PIN1 silencing effectively suppressed the migration and invasion in HLE cells containing mutant TP53 gene. Similarly, Girardini et al have demonstrated that RNAi-mediated knockdown of either mutant TP53 or PIN1 significantly attenuated cell migration and invasion of MDA-MB-231 breast cancer cells containing mutant TP53 (14). Notably, in contrast to PIN1 silencing in HLE cells, PIN1 silencing in HepG2 cells containing wild-type TP53 gene yielded slightly increased migration and invasion. These findings suggest that an interaction between PIN1 and functional TP53 results in cellular 
effects opposite to those occurring in the presence of mutant TP53. High expression of PIN1 is correlated with poor prognosis in several types of cancers, including HCC (21,24-27). However, previous studies did not perform TP53 gene mutation analysis, or details of the TP53 gene status were not provided. The biological behavior of PIN1 according to the context of TP53 gene in HCC remains unclear. We found that RFS was significantly longer in patients with HCC wild-type TP53 with PIN1 expression. Similar to our result, Lill et al have demonstrated that high expression of PIN1 is a good prognostic factor in patients with Merkel cell carcinoma, which shows infrequent TP53 mutation $(22,28)$. Girardini et al have reported that OS is significantly decreased in patients with breast cancer expressing high levels of PIN1 and mutant TP53 compared to that of patients with low PIN1 expression and mutant or wild-type TP53 (14). However, PIN1 expression in combination with TP53 mutation was not found to be a poor prognostic factor in the present study. This discrepancy in the clinical relevance of PIN1 may be partly explained by differences in tumor type and an insufficient number of patients in the present study. Additional investigations with a larger population of HCC patients with simultaneous assessment of the TP53 gene and other cellular partner genes of PIN1 are necessary to determine the combination of PIN1 expression and mutant TP53 gene that serves as a prognostic and predictive tool.

In conclusion, our findings strongly suggest that the PIN1 can exert a conditional tumor promoter or suppressor role depending on the TP53 gene mutation status in HCC. Our results also support the need to evaluate the status of the TP53 gene in the development of therapeutic approaches for targeting PIN1 in HCC patients.

\section{Acknowledgements}

The present study was supported by the National Research Foundation of Korea (NRF) grant funded by the Korean Government (MSIP) (no. 2008-0062279), and fund of Biomedical Research Institute, Chonbuk National University Hospital.

\section{References}

1. Ferlay J, Soerjomataram I, Dikshit R, Eser S, Mathers C, Rebelo M, Parkin DM, Forman D and Bray F: Cancer incidence and mortality worldwide: Sources, methods and major patterns in GLOBOCAN 2012. Int J Cancer 136: E359-E386, 2015.

2. Graf D, Vallböhmer D, Knoefel WT, Kröpil P, Antoch G, Sagir A and Häussinger D: Multimodal treatment of hepatocellular carcinoma. Eur J Intern Med 25: 430-437, 2014.

3. Vousden KH and Prives C: Blinded by the light: The growing complexity of p53. Cell 137: 413-431, 2009.

4. Rivlin N, Brosh R, Oren M and Rotter V: Mutations in the p53 tumor suppressor gene: Important milestones at the various steps of tumorigenesis. Genes Cancer 2: 466-474, 2011.

5. Liu J, Ma Q, Zhang M, Wang X, Zhang D, Li W, Wang F and Wu E: Alterations of TP53 are associated with a poor outcome for patients with hepatocellular carcinoma: Evidence from a systematic review and meta-analysis. Eur J Cancer 48: 2328-2338, 2012.

6. Kim MP, Zhang Y and Lozano G: Mutant p53: Multiple mechanisms define biologic activity in cancer. Front Oncol 5: 249 2015.
7. Dowell SP, Wilson PO, Derias NW, Lane DP and Hall PA Clinical utility of the immunocytochemical detection of p53 protein in cytological specimens. Cancer Res 54: 2914-2918, 1994.

8. Yeh ES and Means AR: PIN1, the cell cycle and cancer. Nat Rev Cancer 7: 381-388, 2007.

9. Bao L, Kimzey A, Sauter G, Sowadski JM, Lu KP and Wang DG: Prevalent overexpression of prolyl isomerase Pin1 in human cancers. Am J Pathol 164: 1727-1737, 2004.

10. Finn G and Lu KP: Phosphorylation-specific prolyl isomerase Pin1 as a new diagnostic and therapeutic target for cancer. Curr Cancer Drug Targets 8: 223-229, 2008.

11. Yeh ES, Lew BO and Means AR: The loss of PIN1 deregulates cyclin $\mathrm{E}$ and sensitizes mouse embryo fibroblasts to genomic instability. J Biol Chem 281: 241-251, 2006.

12. Teng BL, Hacker KE, Chen S, Means AR and Rathmell WK: Tumor suppressive activity of prolyl isomerase Pin1 in renal cell carcinoma. Mol Oncol 5: 465-474, 2011.

13. Mantovani F, Zannini A, Rustighi A and Del Sal G: Interaction of p53 with prolyl isomerases: Healthy and unhealthy relationships. Biochim Biophys Acta 1850: 2048-2060, 2015.

14. Girardini JE, Napoli M, Piazza S, Rustighi A, Marotta C, Radaelli E, Capaci V, Jordan L, Quinlan P, Thompson A, et al: A Pin $1 /$ mutant 553 axis promotes aggressiveness in breast cancer. Cancer Cell 20: 79-91, 2011.

15. Choi HN, Bae JS, Jamiyandorj U, Noh SJ, Park HS, Jang KY, Chung MJ, Kang MJ, Lee DG and Moon WS: Expression and role of SIRT1 in hepatocellular carcinoma. Oncol Rep 26: 503-510, 2011.

16. Sung JJ, Noh SJ, Bae JS, Park HS, Jang KY, Chung MJ and Moon WS: Immunohistochemical expression and clinical significance of suggested stem cell markers in hepatocellular carcinoma. J Pathol Transl Med 50: 52-57, 2016.

17. Bae JS, Noh SJ, Kim KM, Jang KY, Chung MJ, Kim DG and Moon WS: Serum response factor induces epithelial to mesenchymal transition with resistance to sorafenib in hepatocellular carcinoma. Int J Oncol 44: 129-136, 2014.

18. Pang RW, Lee TK, Man K, Poon RT, Fan ST, Kwong YL and Tse E: PIN1 expression contributes to hepatic carcinogenesis. J Pathol 210: 19-25, 2006.

19. Pang R, Yuen J, Yuen MF, Lai CL, Lee TK, Man K, Poon RT, Fan ST, Wong CM, Ng IO, et al: PIN1 overexpression and betacatenin gene mutations are distinct oncogenic events in human hepatocellular carcinoma. Oncogene 23: 4182-4186, 2004.

20. Pang R, Lee TK, Poon RT, Fan ST, Wong KB, Kwong YL and Tse E: Pin1 interacts with a specific serine-proline motif of hepatitis B virus X-protein to enhance hepatocarcinogenesis. Gastroenterology 132: 1088-1103, 2007.

21. Shinoda K, Kuboki S, Shimizu H, Ohtsuka M, Kato A, Yoshitomi H, Furukawa K and Miyazaki M: Pin1 facilitates $\mathrm{NF}-\kappa \mathrm{B}$ activation and promotes tumour progression in human hepatocellular carcinoma. Br J Cancer 113: 1323-1331, 2015.

22. Lill C, Schneider S, Pammer J, Loewe R, Gedlicka W, Houben R, Heiduschka G, Brunner M and Thurnher D: Significant correlation of peptidyl-prolyl isomerase overexpression in Merkel cell carcinoma with overall survival of patients. Head Neck 33: 1294-1300, 2011.

23. Llovet JM, Burroughs A and Bruix J: Hepatocellular carcinoma. Lancet 362: 1907-1917, 2003.

24. He J, Zhou F, Shao K, Hang J, Wang H, Rayburn E, Xiao ZX, Lee SW, Xue Q, Feng XL, et al: Overexpression of Pin1 in non-small cell lung cancer (NSCLC) and its correlation with lymph node metastases. Lung Cancer 56: 51-58, 2007.

25. Jin H, Jiang J, Sun L, Zheng F, Wu C, Peng L, Zhao Y and Wu X: The prolyl isomerase Pin1 is overexpressed in human esophageal cancer. Oncol Lett 2: 1191-1196, 2011.

26. Tan X, Zhou F, Wan J, Hang J, Chen Z, Li B, Zhang C, Shao K, Jiang P, Shi S, et al: Pin1 expression contributes to lung cancer: Prognosis and carcinogenesis. Cancer Biol Ther 9: 111-119, 2010.

27. Matsuura I, Chiang KN, Lai CY, He D, Wang G, Ramkumar R, Uchida T, Ryo A, Lu K and Liu F: Pin1 promotes transforming growth factor-beta-induced migration and invasion. J Biol Chem 285: 1754-1764, 2010.

28. Lill C, Schneider S, Item CB, Loewe R, Houben R, Halbauer D, Heiduschka G, Brunner M and Thurnher D: P53 mutation is a rare event in Merkel cell carcinoma of the head and neck. Eur Arch Otorhinolaryngol 268: 1639-1646, 2011. 